\title{
Obtenção e caracterização de farinha de casca de uva e sua utilização em snack extrusado
}

\author{
Obtention and characterization of grape skin flour and its use in an extruded snack
}

\section{Ana Betine Beutinger Bender ${ }^{1 *}$, Márcia de Mello Luvielmo², Bruno Bianch Loureiro ${ }^{3}$, Caroline Sefrin Speroni ${ }^{4}$, Aline Augusti Boligon ${ }^{5}$, Leila Picolli da Silva ${ }^{3}$, Neidi Garcia Penna ${ }^{4}$}

\author{
'Universidade Federal de Santa Maria (UFSM), Campus Frederico Westphalen, Frederico Westphalen/RS - Brasil \\ 2Universidade Federal do Rio Grande (FURG), Escola de Química e Alimentos, Rio Grande/RS - Brasil \\ ${ }^{3}$ Universidade Federal de Santa Maria (UFSM), Departamento de Zootecnia, Laboratório de Piscicultura, Santa Maria/RS - Brasil \\ ${ }^{4}$ Universidade Federal de Santa Maria (UFSM), Departamento de Tecnologia e Ciência dos Alimentos, Santa Maria/RS - Brasil \\ ${ }^{5}$ Universidade Federal de Santa Maria (UFSM), Departamento de Farmácia Industrial, Laboratório de Fitoquímica, Santa Maria/RS - Brasil
}

\section{*Corresponding Author}

Ana Betine Beutinger Bender, Universidade Federal de Santa Maria (UFSM), Campus Frederico Westphalen, Linha Sete de Setembro, s/n, BR 386, Km 40, CEP: 98400-000, Frederico Westphalen/RS - Brasil, e-mail: betinebender@hotmail.com

Cite as: Obtention and characterization of grape skin flour and its use in an extruded snack. Braz. J. Food Technol., v. 19, e2016010, 2016.

Received: Jan. 01, 2015; Accepted: Jun. 03, 2016

\section{Resumo}

Este estudo propôs caracterizar a farinha de casca de uva (FCU) e o efeito de sua inclusão em snack extrusado no que diz respeito aos parâmetros nutricionais, tecnológicos e sensoriais. Para a obtenção da farinha de casca de uva, foi utilizado bagaço de uva da cultivar Marselan (Vitis vinifera) proveniente do processo de vinificação. O bagaço in natura foi seco em estufa de circulação de ar a $55^{\circ} \mathrm{C}$ por 24 horas. A separação das cascas e sementes foi realizada com auxílio de peneiras de $3 \mathrm{~mm}$ e $2 \mathrm{~mm}$, sendo o resíduo posteriormente moído em micromoinho a 27.000 rpm (partículas $<1 \mathrm{~mm}$ ) e armazenado $\mathrm{a}-18^{\circ} \mathrm{C}$. A farinha de casca de uva obtida foi submetida às análises de composição química (umidade, cinzas, proteína, lipídeos, fibra alimentar total e carboidratos, pH, compostos fenólicos e cor). A FCU foi incluída na formulação de snacks extrusados nas concentrações de $9 \%$ e 18\%, em substituição à farinha de milho, representando 5\% e 10\% de fibra, respectivamente, os quais foram avaliados quanto à cor, textura e aceitação sensorial. Realizaram-se as determinações microbiológicas de coliformes a $45^{\circ} \mathrm{C}$ e Salmonella nas formulações de snack extrusado. Fibra $(58,01 \%)$, carboidratos $(17,62 \%)$ e cinzas (12,46\%) foram os principais constituintes da farinha de casca de uva, a qual teve pH de 3,51 . Resveratrol (6,14 mg. $\left.\mathrm{g}^{-1}\right)$, luteolina $\left(5,16 \mathrm{mg} \cdot \mathrm{g}^{-1}\right)$ e kaempferol $\left(3,01 \mathrm{mg} \cdot \mathrm{g}^{-1}\right)$ foram os compostos fenólicos detectados em maior quantidade na FCU, indicando presença de antioxidantes na farinha. A formulação de snack contendo 9\% (5\% fibra) de FCU apresentou melhores resultados de aceitação com relação aos atributos cor, aroma e textura, comparada à formulação de snack padrão. Pelo enriquecimento nutricional (fibras e fitoquímicos) e por agregar valor ao resíduo agroindustrial descartado pelas vinícolas, a adição de FCU em snacks extrusados é viável e bastante interessante.

Palavras-chave: Bagaço de uva; Compostos fenólicos; Resíduo agroindustrial; Sensorial.

\section{Summary}

This study aimed to characterize grape skin flour (GSF) and the effect of its inclusion in an extruded snack, with regard to the nutritional, technological and sensory parameters. Grape pomace from the cultivar Marselan (Vitis vinifera), obtained during the winemaking process, was used to obtain the grape skin flour. The in natura pomace was dried in a forced air oven at $55^{\circ} \mathrm{C}$ for 24 hours. The skins were separated from the seeds using $2 \mathrm{~mm}$ and $3 \mathrm{~mm}$ sieves, and the skins subsequently ground in a micro mill at $27.000 \mathrm{rpm}$ (particles $<1 \mathrm{~mm}$ ) and stored at $-18^{\circ} \mathrm{C}$. The grape skin flour obtained was submitted to a chemical analysis (moisture, ash, protein, lipids, total dietary fibre, carbohydrates, $\mathrm{pH}$, phenolic compounds and colour). The GSF was included in the formulation of an extruded snack at levels of $9 \%$ and $18 \%$, substituting the corn flour, representing $5 \%$ and $10 \%$ fibre, respectively. The snacks were evaluated for colour, texture and acceptance. A microbiological analysis for coliforms at $45^{\circ} \mathrm{C}$ and Salmonella was carried out on the test formulations. Fibre (58.01\%), ash (12.46\%) and carbohydrates 
(17.62\%) were the main constituents of the grape skin flour, which had a pH value of 3.51 . The phenolic compounds resveratrol $\left(6.14 \mathrm{mg} \cdot \mathrm{g}^{-1}\right)$, luteolin $\left(5.16 \mathrm{mg} . \mathrm{g}^{-1}\right)$ and kaempferol $\left(3.01 \mathrm{mg} . \mathrm{g}^{-1}\right)$ were detected in the largest amounts in the GSF, indicating the presence of antioxidants in the flour. The snack containing 9\% (5\% fibre) of GSF showed the best results for acceptance with respect to the attributes of colour, aroma and texture as compared to the standard snack. The addition of GSF to extruded snacks is feasible and of interest due to its nutritional enrichment (fibre and phytochemicals) and aggregation of value to a byproduct discarded by wineries.

Keywords: Grape pomace; Phenolic compounds; Agroindustrial waste; Sensory evaluation.

\section{Introdução}

A indústria vitivinícola gera volumes substanciais de resíduos orgânicos sólidos, os quais são subaproveitados ou descartados. Estima-se que 73 milhões de toneladas de uvas, principalmente cultivadas como Vitis vinifera, são produzidas mundialmente (EMBRAPA, 2015), das quais o bagaço de uva representa aproximadamente 20\% do volume total (ROCKENBACH et al., 2011a).

Os principais resíduos agroindustriais da vinificação são separados durante as etapas de esmagamento e prensagem das uvas, dos quais somente pequenas quantidades desses resíduos são valorizadas ou aproveitadas (MONRAD et al., 2010). Por sua vez, representam excelentes fontes para aplicações comerciais ainda não exploradas (BARROS, 2011) e o seu reaproveitamento pode contribuir para reduzir impactos ambientais e perdas econômicas, além de representar avanço na manutenção do equilíbrio do meio ambiente. Na literatura científica, há relatos de inclusão de resíduos agroindustriais, tais como farelo de arroz desengordurado, subprodutos do processamento de polpa de fruta e farinha de bagaço de uva em pães (PAZ et al., 2015) e biscoitos (ABUD; NARAIN, 2009; PIOVESANA et al., 2013).

O bagaço de uva é constituído principalmente de cascas, sementes e resíduos de polpa (ZOCCA et al., 2007). Cascas e sementes vêm sendo utilizadas para obtenção de ingredientes funcionais, tais como antioxidantes naturais e suplementos alimentares (MONRAD et al., 2010). As cascas representam, em média, $82 \%$ do peso seco do bagaço, contendo uma grande quantidade de compostos fenólicos, incluindo antocianinas, ácidos hidroxicinâmicos, catequinas e flavonóis (ROCKENBACH et al., 2011a). Além disso, apresentam teores significativos de fibra alimentar (65-80\%), apresentando alto valor nutritivo (LLOBERA; CAÑELLAS, 2008).

A aplicação de ingredientes ricos em fibra alimentar em produtos alimentícios, tais como snacks, é uma ótima alternativa para inclusão destes na dieta. Snacks extrusados são obtidos por extrusão termoplástica, processo térmico de curta duração que ocorre a temperaturas elevadas, em que materiais amiláceos e/ou proteicos umedecidos são plasticizados e cozidos pela combinação de umidade, pressão, temperatura e cisalhamento mecânico (FERREIRA, 2010). As matérias-primas são reestruturadas e criam-se novas formas e texturas, com melhora das propriedades sensoriais e da digestibilidade do produto extrusado (SELANI et al., 2014).

Diante do exposto, o presente estudo propôs caracterizar a farinha de casca de uva e avaliar o efeito da sua inclusão em snack extrusado, no que diz respeito aos parâmetros nutricionais, tecnológicos e sensoriais.

\section{Material e métodos}

Para obtenção da farinha de casca de uva (FCU), foi utilizado bagaço de uva da cultivar Marselan (Vitis vinifera) proveniente do processo de vinificação, coletado em vinícola de Santa Maria/RS, na safra 2013/2014. A amostra foi coletada após o processo fermentativo das uvas (2 a 3 semanas). O bagaço in natura (casca + semente) foi previamente seco em estufa de circulação de ar a $55^{\circ} \mathrm{C}$ por 24 horas, as condições do processo de secagem foram estabelecidas a partir de resultados obtidos em ensaios preliminares. A separação das cascas e sementes foi realizada com o auxílio de peneiras. Primeiramente passou-se o bagaço seco em peneira de $3 \mathrm{~mm}$, com a finalidade de diminuir o tamanho de partícula e, posteriormente, foi utilizada peneira de $2 \mathrm{~mm}$, a fim de separar a casca da semente (semente retida na peneira). Ambos os processos foram realizados por meio de fricção manual. A seguir, o resíduo contendo as cascas foi moído em micromoinho (Marconi ${ }^{\circledR}$, Piracicaba, Brasil, 27.000 rpm, partículas < $1 \mathrm{~mm}$ ) e armazenado em sacos de polietileno de baixa densidade a $-18{ }^{\circ} \mathrm{C}$, até o momento das análises.

Para caracterização química da farinha de casca de uva foram realizadas análises de matéria seca $\left(105^{\circ} \mathrm{C} .12 \mathrm{~h}^{-1}\right)$, cinzas $\left(550^{\circ} \mathrm{C} .6 \mathrm{~h}^{-1}\right)$ e proteína bruta (método de micro Kjeldahl - N ×6,25) conforme Horwitz (2005). O conteúdo lipídico foi analisado pelo método de Bligh e Dyer (1959). O teor de fibra alimentar total foi determinado seguindo metodologia enzimático-gravimétrica (HORWITZ, 2005). Os carboidratos foram estimados por diferença, em base seca. Os resultados da composição química foram expressos em porcentagem (\%) de matéria seca (MS). A amostra também foi avaliada quanto ao $\mathrm{pH}$, seguindo metodologia descrita por Selani et al. (2014).

A cor $\left(L^{*}, a^{*}, b^{*}\right)$ da FCU foi avaliada em colorímetro CM-700d (Minolta Ltda., Osaka, Japão) utilizando cubetas, com iluminante $D_{65}$ ângulo de $10^{\circ}$. Foram verificados os 
parâmetros de luminosidade $L^{*}[0$ (preto) a 100 (branco], $\mathrm{a}^{*}$ [cromaticidade do verde $(-60)$ a vermelho $\left.(+60)\right] \mathrm{e}$ $\mathrm{b}^{*}$ [cromaticidade do azul $(-60)$ a amarelo $\left.(+60)\right]$. Os valores de Hue $\left({ }^{\circ} \mathrm{h}\right)$, que indicam $0^{\circ}=$ vermelho; $90^{\circ}=$ amarelo; $180^{\circ}=$ verde e $270^{\circ}=$ azul, foram obtidos relacionando-se os valores de $a^{*}$ e $b^{*}\left(H u e=\operatorname{tg}^{-1} b / a\right)$. As leituras foram realizadas em diferentes pontos da farinha, totalizando doze medições por tratamento.

A identificação e a quantificação de compostos fenólicos foram realizadas de acordo com o método descrito por Castillo-Muñoz et al. (2010) utilizando-se a técnica de cromatografia líquida de alta eficiência (CLAE) em fase reversa, em equipamento modelo Shimadzu Prominence Auto Sampler (SIL-20A) (Shimadzu, Kyoto, Japão), acoplado a um detector de arranjo de diodos (DAD). Utilizou-se coluna de sílica em fase reversa $\left(C_{18}\right)$, com dimensões de 4,6 mm $\times 150 \mathrm{~mm}$ e tamanho de partícula de $5 \mu \mathrm{m}$. Os solventes utilizados na fase móvel foram: solvente $A$ (acetonitrila/água/ácido fórmico, 3:88:9, v/v/v), solvente B (acetonitrila/água/ácido fórmico, 50:41:9, v/v/v) e solvente $C$ (metanol/água/ácido fórmico, 90:1:9, v/v/v). A taxa de fluxo foi de 0,6 mL. $\mathrm{min}^{-1}$. O gradiente de solventes utilizados foi: 96\% A, 4\% B e 0\% C mantidos por 7 min; $70 \%$ A, 17\% B e 13\% C após 31 min; 50\% A, 30\% B e 20\% C após 14 min; $30 \%$ A, 40\% B e 30\% C após 0,5 min; 0\% A, 50\% B e $50 \% \mathrm{C}$ após 4,5 min e mantidos por 1 min, retornando às condições iniciais após $7 \mathrm{~min}$. A quantificação foi realizada utilizando-se cromatogramas-DAD obtidos em 254 nm para ácido gálico e resveratrol, $280 \mathrm{~nm}$ para catequina, $327 \mathrm{~nm}$ para ácidos cafeico e clorogênico, $365 \mathrm{~nm}$ para quercetina, rutina, kaempferol e luteolina, e $520 \mathrm{~nm}$ para cianidina, através de curvas de calibrações de padrões externos. A identificação dos picos foi realizada pela comparação dos tempos de retenção com os padrões e com os espectros de DAD (200 a $600 \mathrm{~nm}$ ).

Depois de testes preliminares, a farinha de casca de uva foi incluída na formulação de snack extrusado, nas concentrações de $9 \%$ e 18\%, em substituição à farinha de milho, representando $5 \%$ e $10 \%$ de fibra, respectivamente.
Uma formulação controle (sem adição de FCU) também foi preparada (Figura 1). As amostras foram condicionadas para o teor de umidade de $15 \%$. As formulações foram processadas em extrusora laboratorial de rosca simples. Foram adotados os seguintes parâmetros: temperatura de extrusão entre 90 e $100^{\circ} \mathrm{C}$, taxa de alimentação 226,8 g. $\mathrm{min}^{-1}$ e matriz laminar de $4 \mathrm{~mm}$. A temperatura de secagem após o processo de extrusão foi de $50^{\circ} \mathrm{C}$ em estufa com circulação de ar, por 30 minutos. Os extrusados secos foram armazenados em embalagens laminadas (à base de poliéster metalizado) e armazenados em temperatura ambiente, até o momento das análises químicas e sensoriais.

Para avaliação da qualidade microbiológica das formulações teste, foram realizadas as análises de coliformes a $45^{\circ} \mathrm{C}$ e Salmonella. Todas as análises microbiológicas foram realizadas segundo as metodologias propostas por Silva (2007).

A cor $\left(L^{*}, a^{*}, b^{*}\right)$ dos extrusados foi avaliada em colorímetro CM-700d (Minolta Ltda., Osaka, Japão) utilizando cubetas, com iluminante $D_{65}$ - ângulo de $10^{\circ}$. Foram verificados os parâmetros de luminosidade $L^{*}$ [0 (preto) a 100 (branco], $a^{*}$ [cromaticidade do verde $(-60)$ a vermelho $(+60)] \mathrm{e} \mathrm{b}^{*}$ [cromaticidade do azul (-60) a amarelo (+60)]. Os valores de Hue $\left({ }^{\circ} \mathrm{h}\right)$, que indicam $0^{\circ}=$ vermelho; $90^{\circ}=$ amarelo; $180^{\circ}=$ verde e $270^{\circ}=$ azul, foram obtidos relacionando-se os valores de $a^{*} e b^{*}\left(H u e=\operatorname{tg}^{-1} \mathrm{~b} / \mathrm{a}\right)$. Para a análise, os extrusados foram previamente moídos em micromoinho (marca Marconi ${ }^{\circledR}, 27.000$ rpm, partículas $<1 \mathrm{~mm}$ ), seguindo metodologia proposta por Selani et al. (2014). As leituras foram realizadas em diferentes pontos da farinha, totalizando doze medições por tratamento. A análise de textura foi realizada de acordo com Ferreira (2010), utilizando texturômetro TA-XT2 (Stable Micro Systems Ltda. TA.XTPlus, Surrey, Inglaterra), por meio da determinação da força máxima de cisalhamento (N). Utilizou-se probe HDP-BS, velocidade do pré-teste $1,5 \mathrm{~mm} \cdot \mathrm{s}^{-1}$, velocidade do teste $1,0 \mathrm{~mm} \cdot \mathrm{s}^{-1}$, velocidade do pós-teste $10,0 \mathrm{~mm} \cdot \mathrm{s}^{-1} \mathrm{e}$ distância $10 \mathrm{~mm}$. Foram realizadas seis medições por tratamento.

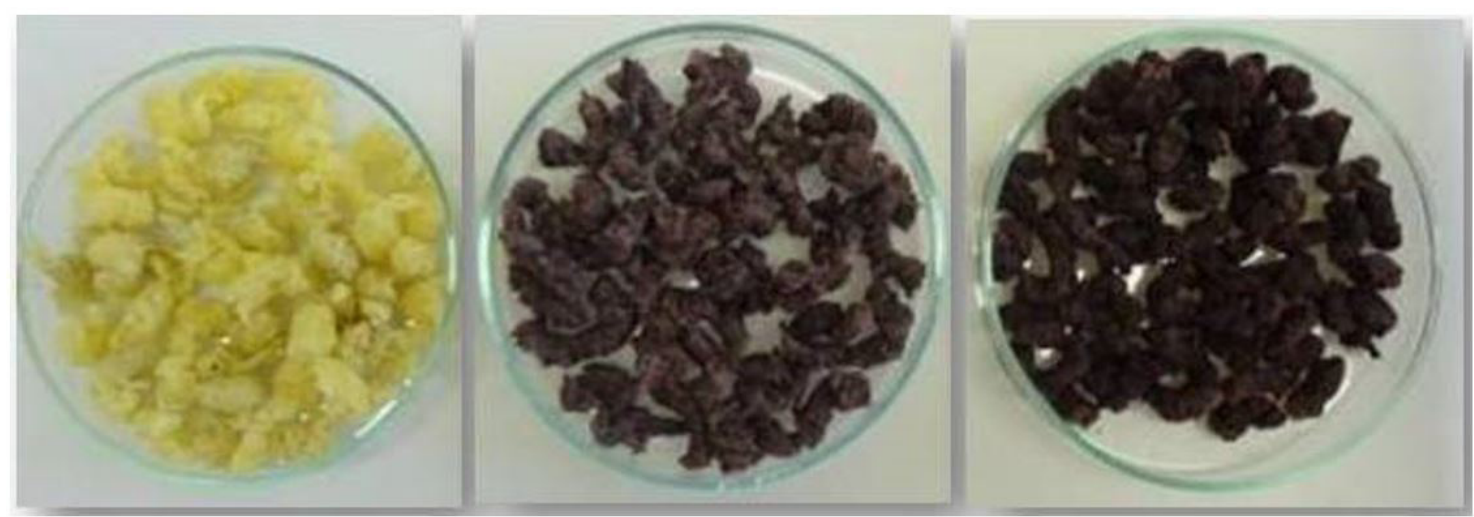

Figura 1. Snacks extrusados - controle, com 9\% e 18\% de inclusão da farinha de casca de uva, respectivamente. 
Para a caracterização dos produtos formulados, procedeu-se à análise sensorial, a qual foi realizada sem que tenha sido alterada a formulação, ou seja, apenas substituiu-se parte da farinha de milho por FCU, não sendo adicionados aditivos quaisquer. $O$ presente estudo foi previamente aprovado pelo Comitê de Ética em Pesquisa na Área da Saúde da Universidade Federal do Rio Grande (CEPAS FURG), sob o n CAAE 39738214.0.0000.5324. As formulações foram submetidas ao Teste Afetivo de Aceitação através de escala hedônica de nove pontos (9 = gostei muito; 1 = desgostei muito). A avaliação sensorial foi realizada no Laboratório de Análise Sensorial e Controle de Qualidade de Alimentos da Escola de Química e Alimentos, Universidade Federal do Rio Grande (FURG), por 60 avaliadores adultos, não treinados, de ambos os sexos (ISO 11136:2012). Avaliou-se cada amostra separadamente, quanto aos atributos cor, aroma, sabor, textura e aceitação global (DUTCOSKY, 2013).

O experimento foi conduzido em delineamento completamente casualizado. Para resultados submetidos à análise estatística, foi utilizada a análise de variância (ANOVA), sendo as médias das amostras comparadas pelo teste de Tukey com nível de $5 \%$ de probabilidade $(p \leq 0,05)$.

\section{Resultados e discussão}

A composição química da farinha de casca de uva (Tabela 1) é constituída de alto teor de fibra alimentar (58\%). Segundo a RDC 54/2012, esta farinha pode ser considerada fonte de fibra, visto que apresenta teor superior ao estabelecido pela Legislação, de $3 \mathrm{~g} / 100 \mathrm{~g}$ (BRASIL, 2012). Resultados semelhantes (51,1\% a 56,3\%) foram relatados por Deng et al. (2011). Depois do conteúdo de fibra, os maiores valores encontrados foram para o teor de carboidratos e cinzas. O conteúdo de carboidratos encontrado no presente estudo está abaixo do relatado por Ferreira (2010).

O teor de umidade da farinha foi de $7,17 \%$, dentro dos padrões exigidos pela RDC 263/2005, que estabelece um teor máximo de umidade de $15 \%$ para farinhas obtidas de frutos e sementes (BRASIL, 2005). A farinha de casca de uva apresentou teor relevante de cinzas (12,46\%), fato decorrente da maior concentração de minerais nas frações externas dos frutos (GONDIM et al., 2005). O conteúdo proteico $(6,78 \%)$ encontrado no presente estudo está abaixo dos valores relatados por Deng et al. (2011), entre 11,26\% e $12,35 \%$ e por Sousa et al. (2014), 8,49\%. Diferenças na composição química de resíduos agroindustriais da vinificação, tais como a casca de uva, são atribuídas a fatores agroclimáticos e de práticas enológicas da região do vinhedo, como sistema de condução da vinha, irrigação, emprego de adubação e estado sanitário das uvas no momento da colheita (IBRAVIN, 2010).

O baixo conteúdo lipídico observado (5,13\%) deve-se à utilização somente das cascas para obtenção da farinha, sendo que o maior conteúdo de lipídeos encontra-se nas sementes, entre $10 \%$ e $16 \%$, dependendo da variedade (LUQUE-RODRIGUEZ et al., 2005).

A FCU apresenta coloração característica da casca de uva tinta, com valores de $L^{*} 39,67 \pm 6,28$, estando próxima ao meio da escala e, de acordo com o ângulo Hue $\left(4,45^{\circ} \pm 1,10\right)$, a coloração aproxima-se do croma vermelho, logo a FCU apresenta croma vermelho com tonalidade escura. A coloração escura de um ingrediente, em alguns casos, limita a sua utilização em produtos alimentícios (SELANI et al., 2014). Walker et al. (2014) relatam que a inclusão de ingredientes com coloração escura em produtos alimentícios vem sendo associada, pelos consumidores, com ingredientes integrais e, portanto, mais saudáveis.

$\mathrm{O}$ valor de $\mathrm{pH}$ de 3,51 \pm 0,02 encontrado neste estudo para a FCU está de acordo com o encontrado por Ferreira (2010), que obteve pH 3,50 em farinha de bagaço de uva, classificando-a como um produto muito ácido. Segundo Selani et al. (2014), a baixa umidade e baixo pH, diminuem o risco de reações enzimáticas, não enzimáticas e contaminação microbiológica.

Através das análises cromatográficas, constatou-se que flavonoides, ácidos fenólicos, antocianinas e taninos estão presentes na FCU (Tabela 2), sendo que os flavonoides resveratrol, luteolina e kaempferol encontram-se em maiores quantidades. Dentre os compostos quantificados, destaca-se o teor de resveratrol $\left(6,15 \mathrm{mg} \mathrm{g}^{-1}\right)$, composto presente em diversas espécies de plantas, sendo que a casca de uva é considerada ótima fonte deste composto (ROCKENBACH et al., 2011b). Neste estudo realizado por Rockenbach et al. (2011b), ao avaliar o conteúdo de compostos fenólicos em cascas de uva provenientes da vinificação de seis cultivares (Pinot Noir, Isabel, Sangiovese, Negro Amaro, Cabernet Sauvignon e Primitivo), não foi detectada a presença de resveratrol, em decorrência da sua transferência para o vinho, ficando abaixo do limite de detecção do método utilizado.

Tabela 1. Composição química da farinha de casca de uva.

\begin{tabular}{cccccc} 
Amostra & Cinzas & Proteína & $\begin{array}{c}\text { Lipídeos } \\
\% \text { na matéria seca }\end{array}$ & FAT & Carboidratos \\
\hline FCU & $12,46 \pm 0,09$ & $6,78 \pm 0,11$ & $5,13 \pm 0,02$ & $58,01 \pm 0,92$ & 17,62 \\
\hline
\end{tabular}

Resultados expressos como média \pm desvio padrão; FCU: Farinha de Casca de Uva; FAT: Fibra Alimentar Total. 
Doshi et al. (2015) encontraram teor de resveratrol de $37,5 \mathrm{mg} . \mathrm{L}^{-1}$ em cascas de uva da cultivar Merlot. Enquanto Sagdic et al. (2011) obtiveram teor de 12,2 e 14,6 $\mu \mathrm{g} \cdot \mathrm{g}^{-1}$ de resveratrol em bagaços de uva das cultivares Gamay e Kalecick karasi, respectivamente, produzidas na Turquia. Em sequência ao estudo, Rockenbach et al. (2011a) relataram teores de 4,02 e 6,40 mg.100 $\mathrm{g}^{-1} \mathrm{de}$ resveratrol em bagaços de uva das cultivares Cabernet Sauvignon e Merlot, respectivamente. Estudos indicam que o resveratrol apresenta ação antimutagênica, antioxidante, anti-inflamatória e antimicrobiana (LORRAIN et al., 2013) e, dessa forma, a inclusão de farinha de casca de uva, fonte deste composto, auxilia na promoção e proteção da saúde.

O segundo composto fenólico em maior quantidade detectado no presente estudo foi a luteolina $\left(5,16 \mathrm{mg} \cdot \mathrm{g}^{-1}\right)$ (Tabela 2). Este flavonoide não foi quantificado em estudo realizado por Sagdic et al. (2011) ao avaliar bagaços de uva de diferentes cultivares de Vitis vinifera da Turquia.

Traços de kaempferol (< 0,05 $\mathrm{mg} \cdot \mathrm{g}^{-1}$ ) foram encontrados em bagaço de uva da cultivar Cabernet Sauvignon em estudo realizado por Ruberto et al. (2007). O composto kaempferol esteve presente em amostras de bagaço de uva cultivadas na Turquia (Gamay e Kalecick karasi), com teores variando entre 432,1 e 713,4 $\mu \mathrm{g} . \mathrm{g}^{-1}$, e também em amostras de bagaço de uva da cultivar
Merlot (15,09 mg.100 g-1) (ROCKENBACH et al., 2011a); teores inferiores foram quantificados no presente estudo (3,01 mg.g $\left.\mathrm{g}^{-1}\right)$. O conteúdo de compostos fenólicos é influenciado por diversos fatores, dentre eles, fatores agroclimáticos e práticas enológicas da região do vinhedo (RADOVANOVIĆ et al., 2010).

Em relação à qualidade microbiológica dos produtos formulados, a RDC 12/2001 (BRASIL, 2001) estabelece, para cereais matinais e produtos extrusados, valores inferiores a 1 UFC. $g^{-1}$ para coliformes a $45^{\circ} \mathrm{C}$. No presente estudo, as contagens ficaram abaixo deste valor e houve ausência de Salmonella, indicando boas condições higiênicas durante o processamento da FCU.

A cor é um fator de qualidade relacionado diretamente com a aceitabilidade de alimentos processados e trata-se de uma propriedade física importante na análise de produtos extrusados. A adição de farinha de casca de uva ocasionou a diminuição da luminosidade em relação à formulação controle (Tabela 3). Esta redução deve-se à adição da FCU, que apresenta croma vermelho com tonalidade escura e também devido às reações de escurecimento, como Maillard (SELANI et al., 2014) e evaporação da água. De acordo com o ângulo Hue, na formulação controle, observou-se coloração amarela intensa (Tabela 3), devido a utilização apenas de farinha de milho nesta formulação. As formulações contendo

Tabela 2. Conteúdo de compostos fenólicos em farinha de casca de uva.

\begin{tabular}{|c|c|c|c|}
\hline Componentes & $\begin{array}{l}\text { Farinha de casca de uva } \\
\qquad \mathrm{mg}^{-\mathrm{g}^{-1}}\end{array}$ & $\begin{array}{c}\text { LOD } \\
\mu g \cdot \mathrm{mL}^{-1}\end{array}$ & $\begin{array}{c}\mathrm{LOQ} \\
\mu \mathrm{g} \cdot \mathrm{mL}^{-1}\end{array}$ \\
\hline \multicolumn{4}{|l|}{ Ácidos fenólicos } \\
\hline Ácido clorogênico & $2,07 \pm 0,01^{e}$ & 0,032 & 0,105 \\
\hline Ácido gálico & $1,15 \pm 0,02^{\dagger}$ & 0,017 & 0,056 \\
\hline Ácido cafeico & $0,21 \pm 0,01^{9}$ & 0,026 & 0,086 \\
\hline \multicolumn{4}{|l|}{ Flavonoides } \\
\hline Luteolina & $5,16 \pm 0,03^{b}$ & 0,021 & 0,070 \\
\hline Kaempferol & $3,01 \pm 0,01^{c}$ & 0,008 & 0,026 \\
\hline Cianidina & $2,95 \pm 0,03^{c}$ & 0,007 & 0,024 \\
\hline Quercetina & $2,25 \pm 0,01^{d}$ & 0,015 & 0,049 \\
\hline Rutina & $1,93 \pm 0,01^{e}$ & 0,013 & 0,041 \\
\hline Quercitrina & $1,87 \pm 0,02^{e}$ & 0,030 & 0,099 \\
\hline Catequina & $1,10 \pm 0,03^{\dagger}$ & 0,009 & 0,034 \\
\hline \multicolumn{4}{|l|}{ Estilbeno } \\
\hline Resveratrol & $6,14 \pm 0,02^{a}$ & 0,027 & 0,083 \\
\hline
\end{tabular}

LOD limite de detecção $\left(\mu \mathrm{g} \cdot \mathrm{mL}^{-1}\right)$; LOQ limite de quantificação $\left(\mu \mathrm{g} \cdot \mathrm{mL}^{-1}\right)$. Resultados expressos como médias \pm desvio padrão. Médias seguidas de letras diferentes diferem entre si pelo teste de Tukey $(p<0,01)$.

Tabela 3. Efeito da inclusão de farinha de casca de uva nos parâmetros tecnológicos dos snacks extrusados.

\begin{tabular}{lcccc}
\multicolumn{1}{c}{ Amostra } & Umidade (\%) & $\mathbf{L}^{*}$ & ${ }^{*}$ Hue & Dureza (g.s) \\
Controle & $8,20 \pm 0,0^{\mathrm{b}}$ & $79,46 \pm 2,15^{\mathrm{a}}$ & $85,81 \pm 0,31^{\mathrm{a}}$ & $944,61 \pm 33,21^{\mathrm{a}}$ \\
$5 \%$ Fibra FCU & $9,14 \pm 0,10^{\mathrm{a}}$ & $50,09 \pm 5,22^{\mathrm{b}}$ & $29,29 \pm 1,58^{\mathrm{b}}$ & $253,13 \pm 17,63^{\mathrm{c}}$ \\
$10 \%$ Fibra FCU & $7,47 \pm 0,06^{\mathrm{c}}$ & $43,77 \pm 5,91^{\mathrm{c}}$ & $16,08 \pm 1,72^{\mathrm{c}}$ & $385,52 \pm 3,81^{\mathrm{b}}$ \\
\hline
\end{tabular}

Resultados expressos como média \pm desvio padrão. Letras diferentes, na mesma coluna, diferem entre si pelo teste de Tukey $(p \leq 0,05)$. 
Obtenção e caracterização de farinha de casca de uva e sua utilização em snack extrusado

Bender, A. B. B. et al.

FCU apresentaram coloração vermelha mais intensa, com ${ }^{\circ}$ Hue abaixo dos $45^{\circ}$, em decorrência de reações de escurecimento envolvendo temperatura e presença de açúcares na farinha (SELANI et al., 2014).

A dureza é um dos fatores que determina a qualidade do alimento pelo consumidor e é desejável que seus valores sejam baixos, porém não há definição de valores aceitáveis para snacks (ALVES; GROSSMANN, 2002). De acordo com os resultados de dureza dos snacks (Tabela 3), verifica-se que houve aumento da dureza, pico máximo de força, com o aumento da adição de FCU. Foi observada diferença estatística entre as formulações teste, indicando que o percentual adicionado interferiu neste parâmetro tecnológico. Esse resultado também foi observado por Altan et al. (2008), no estudo, o aumento na adição de bagaço de tomate ocasionou aumento na dureza dos produtos extrusados elaborados.

Através dos resultados da análise sensorial de aceitação global dos produtos, foram calculados os Índices de Aceitação (IA). Para as formulações controle e com $5 \%$ de fibra da FCU, foram obtidos $55,1 \%$ e, para a formulação teste com $10 \%$ fibra FCU, 45,1\%, valores abaixo do IA recomendado de $70 \%$ para lançamento de novos produtos no mercado (DUTCOSKY, 2013). Porém, observando-se mais atentamente a distribuição desses resultados, verifica-se, através das análises de aceitação sensorial (Figura 2), que para os atributos cor, aroma e textura a formulação com 5\% de fibra FCU apresentou maiores médias de aceitação e maiores percentuais na faixa de notas superiores ou iguais a 7 . Observando a Tabela 4, em relação ao atributo cor, podemos verificar que essa formulação atingiu a maior média de aceitação para esse atributo, obtendo melhor desempenho que a própria formulação padrão, a qual possui a cor característica do milho (Figura 2A). Para o atributo aroma (Figura 2B), $28 \%$ dos avaliadores atribuíram notas maiores ou iguais a 7 à formulação 5\% fibra FCU e os maiores escores de aceitação, para esse atributo, foram atribuídos para a referida formulação. Já para o atributo textura, 44\% dos julgadores atribuíram notas maiores ou iguais a 7 para a formulação 5\% fibra FCU (Figura 2C). Em estudo realizado por Makila et al. (2014), ao avaliar a aceitação sensorial

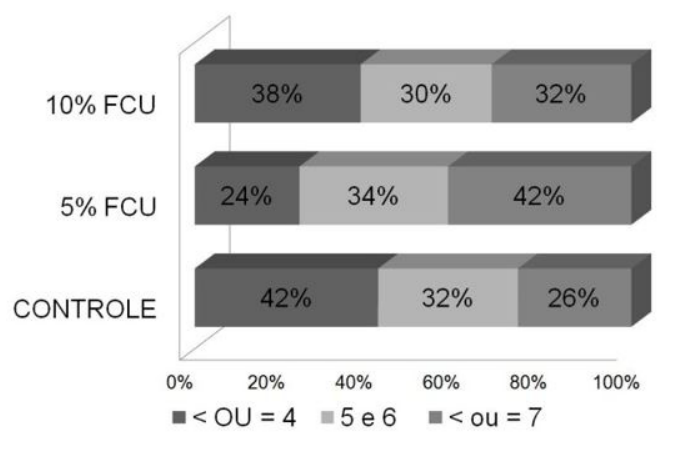

A

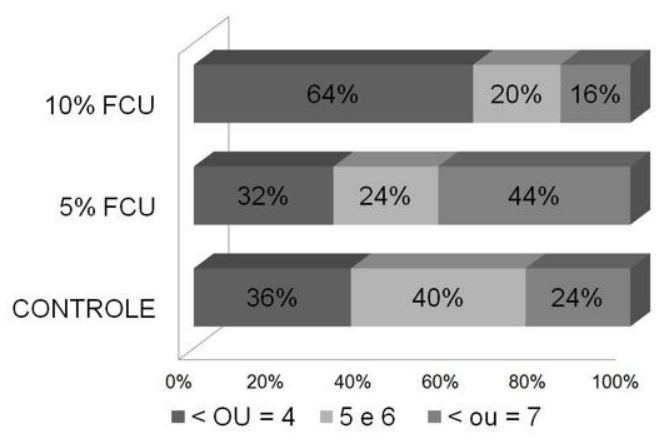

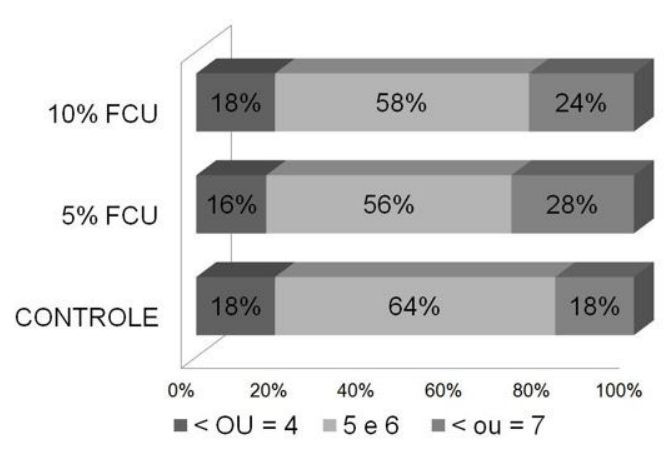

B

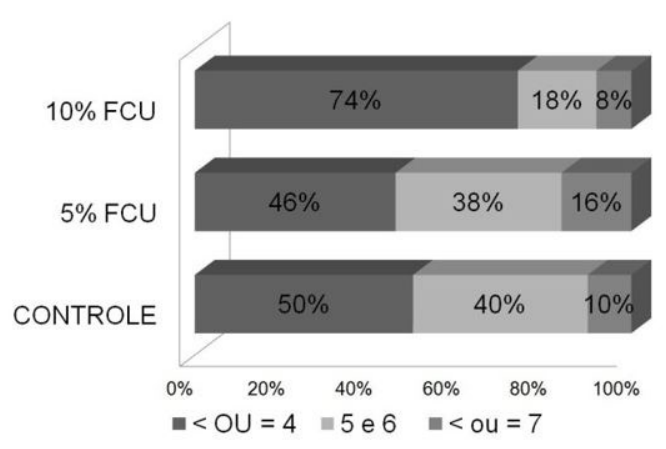

D

Figura 2. Classificação dos escores hedônicos para os atributos cor (A); aroma (B); textura (C) e sabor (D).

Tabela 4. Escores médios para avaliação de aceitação sensorial de snacks extrusados com adição de farinha de casca de uva.

\begin{tabular}{lccccc}
\multicolumn{1}{c}{ Amostra } & Cor & Aroma & Sabor & Textura & Aceitação Global \\
Controle & $5,04^{\mathrm{b}}$ & $5,14^{\mathrm{b}}$ & $4,58^{\mathrm{a}}$ & $5,36^{\mathrm{b}}$ & $4,96^{\mathrm{a}}$ \\
$5 \%$ Fibra FCU & $5,74^{\mathrm{a}}$ & $5,58^{\mathrm{a}}$ & $4,44^{\mathrm{a}}$ & $5,74^{\mathrm{a}}$ & $4,96^{\mathrm{a}}$ \\
$10 \%$ Fibra FCU & $5,20^{\mathrm{b}}$ & $5,26^{\mathrm{b}}$ & $3,68^{\mathrm{b}}$ & $4,10^{\mathrm{c}}$ & $4,06^{\mathrm{b}}$ \\
\hline
\end{tabular}

Letras diferentes, na mesma coluna, diferem entre si pelo teste de Tukey $(p \leq 0,05)$. FCU: farinha de casca de uva. 
de snacks extrusados formulados com adição de resíduo do processamento de suco de groselha com tratamento enzimático e sem tratamento enzimático, observaram-se notas entre 3,7 e 5,7 e 6,1 e 6,4, respectivamente.

O atributo sabor foi o que apresentou menores percentuais de aceitação na escala maior ou igual a 7 , apenas $16 \%$ dos avaliadores atribuíram estas notas a este parâmetro, para a formulação 5\% fibra FCU. Com o aumento da adição de FCU, observou-se diminuição do percentual de aceitação deste parâmetro (apenas 8\% dos avaliadores atribuíram notas iguais ou superiores a 7 para a formulação 10\% fibra FCU) (Figura 2D). Ferreira (2010), ao avaliar sensorialmente snacks extrusados formulados com adição de farinha de bagaço de uva, relatou menores escores (entre 3,42 e 5,25) para a formulação com maior proporção de bagaço (16\% de adição). Observando a Tabela 4, verifica-se que a formulação 5\% fibra FCU não diferiu estatisticamente $(p \leq 0,05)$ da formulação controle.

Em relação à aceitação global, observou-se que o extrusado desenvolvido com adição de $5 \%$ de fibra da FCU apresentou-se superior à formulação controle e à formulação teste com 10\% de fibra da FCU com relação aos parâmetros de cor, aroma e textura, não diferindo da formulação controle quanto ao atributo sabor ( $p \leq 0,05)$.

Considerando que os produtos foram apenas caracterizados, ou seja, que se realizou apenas substituição da farinha de milho por FCU e não se realizou melhoramento da formulação, os resultados foram considerados satisfatórios devido à maior aceitação do produto com adição de FCU correspondente a $5 \%$ de fibra da FCU quanto aos atributos avaliados. Sugere-se a necessidade de revisão de formulação para novos testes considerando os escores atribuídos às amostras e índices de aceitabilidade.

Em estudo realizado por Trombini et al. (2013), os resultados obtidos na análise sensorial global dos produtos extrusados de misturas de farinha de maracujá e fécula de mandioca evidenciaram que $46 \%$ dos provadores gostaram regularmente (valor 7), 20\% dos provadores gostaram muito (valor 8 ), $6 \%$ dos provadores gostaram extremamente (valor 9), 24\% dos provadores gostaram ligeiramente (valor 6), e apenas $4 \%$ dos provadores desgostaram ligeiramente (valor 4). Porém, deve-se destacar que neste estudo os produtos extrusados foram saborizados, fator que pode ter auxiliado na aceitação dos produtos. No presente estudo, a análise sensorial foi realizada para a caracterização dos produtos e, por isso, não se procedeu à saborização.

Yagci e Gogus (2008) obtiveram notas entre 3,6 e 6,6 para aceitação global de snacks extrusados formulados com adição de farinha de avelã parcialmente desengordurada, sendo que as menores notas (3,6 a 4,6) foram atribuídas à formulação contendo maior percentual de adição desta farinha.
A dificuldade de aceitação de produtos extrusados desenvolvidos com ingredientes para enriquecimento nutricional já foi relatada por outros pesquisadores. Ferreira (2010), ao avaliar sensorialmente extrusados formulados com adição de farinha de bagaço de uva, relatou menores escores para a formulação com maior proporção de bagaço, em que os avaliadores atribuíram notas entre 3,71 e 5,79 para as formulações, sendo que a formulação mais aceita foi a de menor substituição de farinha. Nesse mesmo estudo, a textura da formulação controle foi a mais aceita pelos avaliadores (FERREIRA, 2010), diferindo do presente estudo em que a formulação teste com $5 \%$ de fibra foi significativamente mais aceita quanto ao atributo textura.

\section{Conclusão}

A farinha de casca de uva apresenta teores relevantes de fibra, carboidratos e cinzas em sua composição. A adição de farinha de casca de uva em snacks extrusados torna-se viável no que diz respeito aos parâmetros tecnológicos avaliados. Em relação à análise sensorial, a formulação contendo FCU correspondente a 5\% de fibra apresentou resultados satisfatórios quanto aos atributos avaliados, principalmente textura e cor, as quais obtiveram valores maiores de aceitação em relação à amostra controle. Porém, para os parâmetros de aroma e sabor, verifica-se a necessidade de aprimoramento da formulação, visto que apresentaram menores percentuais de aceitação.

Além disso, observou-se a presença de significativas quantidades de resveratrol, luteolina e kaempferol, compostos antioxidantes com importantes propriedades biológicas, farmacêuticas e medicinais, tornando relevantes estudos complementares envolvendo a avaliação da estabilidade destes compostos após o processamento deste tipo de produto.

\section{Referências}

ABUD, A. K. S.; NARAIN, N. Incorporação da farinha de resíduo do processamento de polpa de fruta em biscoitos: uma alternativa de combate ao desperdício. Brazilian Journal of Food Technology, Campinas, v. 12, n. 4, p. 257-265, 2009. http://dx.doi.org/10.4260/BJFT2009800900020.

ALTAN, A.; MCCARTHY, K. L.; MASKAN, M. Evaluation of snack foods from barley-tomato pomace blends by extrusion processing. Journal of Food Engineering, Essex, v. 84, n. 2, p. 231-242, 2008. http://dx.doi.org/10.1016/j.jfoodeng.2007.05.014.

ALVES, R. M. L.; GROSSMANN, M. V. E. Parâmetros de extrusão para produção de "snacks" de farinha de cará (Dioscorea alata). Ciência e Tecnologia de Alimentos, Campinas, v. 22, n. 1, p. 32-38, 2002. http://dx.doi.org/10.1590/S0101-20612002000100006.

BARROS, Z. M. P. Cascas de frutas tropicais como fonte de antioxidantes para enriquecimento de suco pronto. 2011. 85 
Obtenção e caracterização de farinha de casca de uva e sua utilização em snack extrusado

Bender, A. B. B. et al.

f. Dissertação (Mestrado em Ciência e Tecnologia de Alimentos)Escola Superior de Agricultura "Luiz de Queiroz", Universidade de São Paulo, Piracicaba, 2011.

BLIGH, E. G.; DYER, W. J. A rapid method of total lipid extraction and purification. Canadian Journal of Biochemistry and Physiology, Ottawa, v. 37, n. 8, p. 911-917, 1959. http://dx.doi. org/10.1139/059-099. PMid:13671378.

BRASIL. Ministério da Saúde. Agência Nacional de Vigilância Sanitária. Resolução n 12, de 02 de janeiro de 2001. Regulamento técnico sobre os padrões microbiológicos para alimentos. Diário Oficial [da] República Federativa do Brasil, Brasília, DF, 10 jan. 2001. Seção 1.

BRASIL. Ministério da Saúde. Agência Nacional de Vigilância Sanitária. Resolução nº 263, de 22 de setembro de 2005. Dispõe sobre o regulamento técnico para produtos de cereais, amidos, farinhas e farelos. Diário Oficial [da] República Federativa do Brasil, Brasília, DF, 22 set. 2005. Seção 1. Disponível em: <http://portal.anvisa.gov.br/wps/wcm/connect/b683960047457 a8b8736d73fbc4c6735/RDC_273_2005.pdf?MOD=AJPERES>. Acesso em 20 jul. 2015.

BRASIL. Ministério da Saúde. Agência Nacional de Vigilância Sanitária. Resolução n 54, de 12 novembro de 2012. Regulamento técnico Mercosul sobre informação nutricional complementar. Diário Oficial [da] República Federativa do Brasil, Brasília, DF. 12 nov. 2012.

CASTILLO-MUÑOZ, N.; GÓMEZ-ALONSO, S.; GARCÍA-ROMERO, E.; HERMOSÍN-GUTIÉRREZ, I. Flavonol profiles of Vitis vinifera White grape cultivars. Journal of Food Composition and Analysis, San Diego, v. 23, n. 7, p. 699-705, 2010. http://dx. doi. org/10.1016/j.jfca.2010.03.017

DENG, Q.; PENNER, M. H.; ZHAO, Y. Chemical composition of dietary fiber and polyphenols of five different varieties of wine grape pomace skins. Food Research International, Oxford, v. 44, n. 9, p. 2712-2720, 2011. http://dx.doi.org/10.1016/j. foodres.2011.05.026.

DOSHI, P.; ADSULE, P.; BANERJEE, K.; OULKAR, D. Phenolic compounds, antioxidant activity and insulinotropic effect of extracts prepared from grape (Vitis vinifera $L$ ) byproducts. Journal of Food Science and Technology, Mysore, v. 52, n. 1, p. 181-190, 2015. http://dx.doi.org/10.1007/s13197-013-0991-1. PMid:25593367.

DUTCOSKY, S. D. Análise sensorial de alimentos. 4. ed. Curitiba: Editora Universitária Champagnat, 2013. 531 p.

EMPRESA BRASILEIRA DE PESQUISA AGROPECUÁRIA EMBRAPA. Nota técnica: um balanço da Vitivinicultura Mundial em 2014. Brasília: Embrapa, 2015. Disponível em: <https://www. embrapa.br/busca-de-noticias/-/noticia/4007952/nota-tecnica---um-balanco-da-vitivinicultura-mundial-em-2014>. Acesso em: 30 ago. 2015.
FERREIRA, L. F. D. Obtenção e caracterização de farinha de bagaço de uva e sua utilização em cereais matinais expandidos. 2010. 135 f. Tese (Doutorado em Ciência e Tecnologia de Alimentos)-Universidade Federal de Viçosa, Viçosa, 2010.

GONDIM, J. A. M.; MOURA, M. F. V.; DANTAS, A. S.; MEDEIROS, R. L.; SANTOS, K. M. Composição centesimal e de minerais em cascas de frutas. Ciência e Tecnologia de Alimentos, Campinas, v. 25 , n. 4 , p. 825-827, 2005. http://dx.doi.org/10.1590/S010120612005000400032.

HORWITZ, W. (Ed.). Official methods of analysis of the Association of Official Analytical Chemists. 17th. ed. Arlington: AOAC, 2005.

INSTITUTO BRASILEIRO DE VINHOS - IBRAVIN. A vitivinicultura brasileira. Bento Gonçalves: IBRAVIN, 2010. Disponível em: $<$ http://www.ibravin.org.br/brasilvitivinicola.php>. Acesso em: 20 jul. 2015.

LLOBERA, A.; CAÑELLAS, J. Antioxidant activity and dietary fibre of Prensal Blanc white grape (Vitis vinifera) by-products. International Journal of Food Science \& Technology, Oxford, v. 43, n. 11, p. 1953-1959, 2008. http://dx.doi.org/10.1111/j.13652621.2008.01798.x.

LORRAIN, B.; KY, I.; PECHAMAT, L.; TEISSEDRE, P. L. Evolution of analysis of polyphenols from grapes, wines, and extracts. Molecules, Basel, v. 18, n. 1, p. 1076-1100, 2013. http://dx.doi. org/10.3390/molecules18011076. PMid:23325097.

LUQUE-RODRIGUEZ, J. M.; LUQUE DE CASTRO, M. D.; PEREZJUAN, P. Extraction of fatty acids from by superheated hexane. Talanta, Seattle, v. 68, n. 1, p. 126-130, 2005. http://dx.doi. org/10.1016/j.talanta.2005.04.054. PMid:18970294.

MAKILA, L.; LAAKSONEN, O.; DIAZ, J. M. R.; VAHVASELKA, M.; MYLLYMAKI, O.; LEHTOMAKI, I.; LAAKSO, S.; JAHREIS, G.; JOUPPILA, K.; LARMO, P.; YANG, B.; KALLIO, H. Exploiting blackburrant juice press residue in extruded snacks. LWT - Food Science and Technology, Campinas, v. 57, p. 618-627, 2014.

MONRAD, J. K.; HOWARD, L. R.; KING, J. W.; SRINIVAS, K.; MAUROMOUSTAKOS, A. Subcritical solvent extraction of anthocyanin from dried red grape pomace. Journal of Agricultural and Food Chemistry, Washington, v. 58, n. 5, p. 2862-2868, 2010. http:// dx.doi.org/10.1021/jf904087n. PMid:20148515.

PAZ, M. F.; MARQUES, R. V.; SCHUMANN, C.; CORRÊA, L. B.; CORRÊA, É. K. Características tecnológicas de pães elaborados com farelo de arroz desengordurado. Brazilian Journal of Food Technology, Campinas, v. 18, n. 2, p. 128-136, 2015. http:// dx.doi.org/10.1590/1981-6723.6014.

PIOVESANA, A.; BUENO, M. M.; KLAJN, V. M. Elaboração e aceitabilidade de biscoitos enriquecidos com aveia e farinha de bagaço de uva. Brazilian Journal of Food Technology, Campinas, v. 16, n. 1, p. 68-72, 2013. http://dx.doi.org/10.1590/ S1981-67232013005000007. 
Obtenção e caracterização de farinha de casca de uva e sua utilização em snack extrusado

Bender, A. B. B. et al.

RADOVANOVIĆ, B. C.; RADOVANOVIĆ, A. N.; SOUQUET, J. $M$. Phenolic profile and free radical-scavenging activity of Cabernet Sauvignon wines of different geographical origins from the Balkan region. Journal of the Science of Food and Agriculture, London, v. 90, n. 14, p. 2455-2461, 2010. http:// dx.doi.org/10.1002/jsfa.4106. PMid:20648551.

ROCKENBACH, I. I.; GONZAGA, L. V.; RIZELIO, V. M.; GONÇALVES, A. E. S. S.; GENOVESE, M. I.; FETT, R. Phenolic compounds and antioxidant activity of seed and skin extracts of red grape (Vitis vinifera and Vitis labrusca) pomace from Brazilian winemaking. Food Research International, Oxford, v. 44, n. 4, p. 897-901, 2011b. http://dx.doi.org/10.1016/j.foodres.2011.01.049.

ROCKENBACH, I. I.; RODRIGUES, E.; GONZAGA, L. V.; CALIARI, V.; GENOVESE, M. I.; GONÇALVES, A. E. S. S.; FETT, R. Phenolic compounds content and antioxidant activity in pomace from selected red grapes (Vitis vinifera L. and Vitis labrusca L.) widely produced in Brazil. Food Chemistry, London, v. 127, n. 1, p. 174-179, 2011a. http://dx.doi.org/10.1016/j.foodchem.2010.12.137.

RUBERTO, G.; RENDA, A.; DAQUINO, C.; AMICO, V.; SPATAFORA, C.; TRINGALI, C.; TOMMASI, N. Polyphenol constituents and antioxidant activity of grape pomace extracts from five Sicilian red grape cultivars. Food Chemistry, London, v. 100, n. 1, p. 203-210, 2007. http://dx.doi.org/10.1016/j.foodchem.2005.09.041.

SAGDIC, O.; OZTURK, I.; OZKAN, G.; YETIM, H.; EKICI, L.; YILMAZ, M. T. RP-HPLC-DAD analysis of phenolic compounds in pomace extracts from five grape cultivars: evaluation of their antioxidant, antiradical and antifungal activities in orange and apple juices. Food Chemistry, London, v. 126, n. 4, p. 17491758, 2011. http://dx.doi.org/10.1016/j.foodchem.2010.12.075. PMid:25213954.

SELANI, M. M.; BRAZACA, S. G. C.; DIAS, C. T. S.; RATNAYAKE, W. S.; FLORES, R. A.; BIANCHINI, A. Characterization and potential application of pineapple pomace in an extruded product for fibre enhancement. Food Chemistry, London, v. 163, p. 23-30, 2014. http://dx.doi.org/10.1016/j.foodchem.2014.04.076. PMid:24912691.

SILVA, N. Manual de métodos de análise microbiológica de alimentos. 3. ed. São Paulo: Livraria Varela, 2007. 552 p.

SOUSA, E. C.; UCHÔA-THOMAZ, A. M. A.; CARIOCA, J. O. B.; MORAIS, S. M.; LIMA, A.; MARTINS, C. G.; ALEXANDRINO, C. D.; FERREIRA, P. A. T.; RODRIGUES, A. L. M.; RODRIGUES, S. P.; SILVA, J. N.; RODRIGUES, L. L. Chemical composition and bioactive compounds of grape pomace (Vitis vinifera L.), Benitaka variety, grown in the semiarid region of Northeast Brazil. Food Science and Technology, Campinas, v. 34, n. 1, p. 135-142, 2014. http://dx.doi.org/10.1590/S0101-20612014000100020.

TROMBINI, F. R. M.; LEONEL, M.; MISCHAN, M. M. Características físicas, reológicas e sensorial de produtos extrusados de misturas de farinha de maracujá e fécula de mandioca. Ciência Rural, Santa Maria, v. 43, n. 10, p. 1885-1891, 2013. http://dx.doi. org/10.1590/S0103-84782013005000115.

WALKER, R.; TSENG, A.; CAVENDER, G.; ROSS, A.; ZHAO, $Y$. Physicochemical, nutritional, and sensory qualities of wine grape pomace fortified baked goods. Journal of Food Science, Champaign, v. 79, n. 9, p. 1811-1822, 2014. http://dx.doi. org/10.1111/1750-3841.12554. PMid:25102950.

YAGCI, S.; GOGUS, F. Response surface methodology for evaluation of physical and functional properties of extruded snack foods developed from food-by-produtcs. Journal of Food Engineering, Essex, v. 86, n. 1, p. 122-132, 2008. http://dx.doi. org/10.1016/j.jfoodeng.2007.09.018.

ZOCCA, F.; LOMOLINO, G.; CURIONI, A.; SPETTOLI, P.; LANTE, A. Detection of pectinmethylesterase activity in presence of methanol during grape pomace storage. Food Chemistry, London, v. 102, n. 1, p. 59-65, 2007. http://dx.doi.org/10.1016/j. foodchem.2006.01.061. 